\title{
An Overview of Various Carriers for siRNA Delivery
}

\author{
Alleanna Raye Marquez¹, Chikezie O. Madu², Yi Lu ${ }^{3 凶}$ \\ 1. Department of Biology and Advanced Placement Biology, White Station High School, Memphis, Tennessee 38117. USA. sb108vg@gmail.com \\ 2. Departments of Biology and Advanced Placement Biology, White Station High School, Memphis, Tennessee 38117. USA. maduco@scsk12.org \\ 3. Departments of Pathology and Laboratory Medicine, University of Tennessee Health Science Center, Memphis, TN 38163, USA. ylu@uthsc.edu \\ $\square$ Corresponding author: Yi Lu, Ph.D., Department of Pathology and Laboratory Medicine, University of Tennessee Health Science Center, Cancer Research \\ Building, Room 258, 19 South Manassas Street, Memphis, TN 38163 (USA). Tel.: (901) 448-5436; Fax: (901) 448-5496; E-mail: ylu@uthsc.edu \\ (C) Ivyspring International Publisher. This is an open access article distributed under the terms of the Creative Commons Attribution (CC BY-NC) license \\ (https://creativecommons.org/licenses/by-nc/4.0/). See http://ivyspring.com/terms for full terms and conditions.
}

Received: 2018.02.27; Accepted: 2018.05.07; Published: 2018.06.19

\begin{abstract}
RNA interference through the use of short interfering molecules known as short interfering RNA (siRNA) has the potential to greatly advance research in treatments for many diseases because it has the ability to silence the expression of specific genes by helping degrade target mRNA. However, challenges to siRNA delivery have made the development of safe and effective delivery systems paramount in siRNA research. Various types of delivery systems have been proposed and investigated for siRNA delivery and therapy. Although viral vectors have been established to be the most effective in delivering siRNA molecules, they also raise many concerns over biosafety, especially concerning immunogenicity. Therefore, many researchers have begun to investigate and study non-viral vectors. Non-viral vectors are studied because they are typically considered to be safer than viral vectors albeit less efficient as well. The three general non-viral vectors that have been studied for siRNA delivery are lipid-based, non-lipid organic-based, and non-lipid inorganic-based carriers. Within those general parameters of non-viral vector classification are subtypes that are each unique with their own characteristic benefits and downsides. Many of these carriers, as well as even naked siRNA, do have the potential to be modified so that siRNA delivery could be further enhanced with benefits such as greater stability and duration. Researchers still must be wary with alterations as to not interfere with siRNA function. Currently, widespread siRNA therapeutics are still out of reach, but as more advancements in siRNA research including research on their delivery mechanisms are established, the goal of integrating siRNA therapy into the treatment of a multitude of diseases becomes increasingly more of a possibility. Researchers are currently investigating how siRNA can be used to not just treat cancer but ocular and neurodegenerative diseases as well as many others. There are still many obstacles to face and overcome before siRNA therapy can be implemented into the treatment of many diseases, and more research must still be conducted concerning siRNA delivery systems. Many advancements pertaining to siRNA carriers have been made, and many more are likely on their way.
\end{abstract}

Key words: RNA interference, siRNA therapy, siRNA carriers, viral vectors, non-viral vectors

\section{Introduction}

Processed mRNA can be regulated once in the cytoplasm by a mechanism known as RNA interference (RNAi). RNAi regulates the expression of genes by degrading mRNA and can be driven by RNA molecules such as short interfering RNA (siRNA) and microRNA (miRNA). However, whereas miRNAs can inhibit the expression of multiple mRNA targets, siRNAs target and inhibit a specific mRNA target. $[1,2]$

In mammals, the production of siRNA occurs when an endonuclease known as Dicer processes double-stranded RNA (dsRNA) that is introduced into the cell. Dicer, which comes from the ribonuclease III family, cleaves exogenously introduced dsRNA to generate siRNA composed of about 21-23 nucleotides, a 3' end with a dinucleotide 
overhang, and a 5' end containing a monophosphate group. $[1,3]$ This processing by Dicer is necessary for siRNA to properly interact with the RNA-Induced Silencing Complex (RISC). (Figure 3) RISC further cleaves the siRNA to where only the antisense strand remains with the complex, which guides RISC to the specific target mRNA that it complements. [1,4] The component of RISC that is most responsible for cleavage of the sense strand and the degradation of the target mRNA molecule is the Argonaute 2 protein (AGO2). AGO2 contains the three domains that interact with the antisense strand: the MID, PIWI, and PAZ domains. The MID and PIWI domains of AGO2 interact with the monophosphate group at the $5^{\prime}$ end of the siRNA molecule while the PAZ domain interacts with the $3^{\prime}$ dinucleotide overhang. Consequently, the seed region of the antisense strand is opened up for binding to the target mRNA. (Figure 2) $[2,3]$ Additionally, the siRNA can be used by RISC more than once, meaning that the siRNA is not degraded as soon as one target mRNA molecule is degraded by the complex. [4]

The regulation of mRNA by siRNA is one pathway that functions in the RNAi, but it differs from other methods of RNAi due to the fact that siRNA binds only to mRNA that is fully or almost fully complementary to it. Researchers have taken advantage of this aspect of gene regulation by siRNA, allowing them to research the function of a specific gene. [5] Furthermore, researchers are currently exploring how siRNA can be used in both cancer detection and cancer therapy and how it can specifically inhibit oncogene expression and cancer-causing pathways within cells. [6] Additional research with siRNAs is being conducted for the treatment of viral infections and genetic disorders. [7] Further research on siRNAs is being investigated for the development of new drugs as siRNAs can target the expression of any gene, which will help overcome the limitations that restrict the efficiency of small drug molecules and protein-based drugs that are in current use. [1]

Although there are many potential benefits that siRNA has to offer the medical world, certain challenges must be overcome before their use in treatments can become widespread. One challenge to the use of siRNA as a method of therapy for cancers or other disorders is the development of effective and safe delivery systems for it. Delivery systems for siRNA must be efficient, must be able to target the appropriate body cells, and must not harm the patient. Currently, options for delivery systems range from viral vectors to non-viral vectors; however, there are advantages and disadvantages to each. For each siRNA delivery system, researchers are looking for one that has the ability to circulate within the blood for an effective duration, the ability to reach target tissues, and high chances for the siRNA to be taken up by the cells. [15]

\section{Viral Vectors}

Viral vectors are being researched as methods of delivery because of their high efficiency in delivering nucleic acids into cells. Lentiviruses (LVs), adenoviruses (AVs), and adeno-associated viruses (AAVs) are the primary viral vectors being tested and studied. [8]

$\mathrm{LV}$ vectors are attractive for researchers due to their relatively low cytotoxicity and reportedly low immunogenicity; however, AV vectors do have a high immunogenicity, and this high potential to provoke an immune response has made using AV vectors in research challenging. Furthermore, AV vectors can be problematic to apply to clinical trials due to the high probability of encountering pre-existing immunity in humans to AVs. $[9,10]$ AAV vectors are attractive to researchers as they are nonpathogenic, and although they only have the capacity to contain about $4.7 \mathrm{~kb}$ of genetic material, this should not be a problem in the context of RNAi as these vectors would only need to deliver smaller-sized material into cells. However, they face the same challenge as $\mathrm{AV}$ vectors due to similar chances of encountering pre-immune patients. $[11,12]$ Additionally, although LV vectors do pose many benefits as a vector, they still pose risks and challenges such as potential insertional mutagenesis, and even though they have the capacity to carry $8 \mathrm{~kb}$, they potentially face issues in their distribution with their larger size; they also face challenges in production due to their complexity. [13] Although viral vectors are generally advantageous in efficiency, they raise many concerns pertaining to biosafety; thus, other potential vectors for delivery of siRNA into cells are being explored.

\section{Non-viral Vectors}

Due to safety concerns over viral vectors, much research has been poured into advancing non-viral vectors, which compared to viral vectors are easier to produce and safer to use within the human body. Non-viral vectors are particles that are synthetically produced that have the ability to bind to their respective cassette and deliver them successfully to the target site. One drawback is that these types of vectors are generally less efficient when compared to their viral counterparts. [14] Characteristics that non-viral vectors should have are biocompatibility, biodegradability, non-toxicity, stability, the ability to perform endosomal/lysosomal escape, the ability to protect siRNA, and ease of production. [26] Three 
general categories for non-viral vectors are lipid-based vectors, non-lipid organic-based vectors, and non-lipid inorganic-based vectors.

\section{Lipid-based Vectors}

Lipid-based vectors have attracted researchers because of the ability of lipids to spontaneously form bilayers due to their amphipathic properties. Because lipids and phospholipids are two major molecules that comprise cellular membranes, lipid-based siRNA carriers have the natural capacity to interact favorably with these cellular membranes for the uptake of siRNA. Currently, there are generally four prospective lipid-based vectors for siRNA delivery: lipoplexes, lipopolyplexes, stable nucleic-acid-lipid particles (SNALPs), and membrane/core nanoparticles (MCNPs). $[4,15,16]$ The use of lipid-based carriers is further beneficial to research on siRNA delivery because it has been demonstrated that encapsulating RNA with lipids not only decreases the degradation rates of the RNA but also increases cellular uptake of the nucleic acid material. [19] However, even though there have been many advances in developing lipid-based carriers for delivery, their introduction and application into clinical practice has been slow partly due to problems in pharmaceutical manufacturing as well as regulations set out by the government. [23]

\section{Lipoplexes}

Lipoplexes are composed of multiple bilayers composed of cationic lipids and are simply formed by merely combining an appropriate ratio of cationic liposomes to siRNAs. The siRNA-coated liposomes are then surrounded by ruptured lipid membranes. [15] However, cationic liposomes can be disadvantageous because of their potentially high cytotoxicity. [17,22] The use of neutral lipids has thus been investigated, and studies have demonstrated that they are more biocompatible and have superior pharmacokinetics when compared to their cationic counterparts. Entrapment efficiency of siRNA does decrease with neutral lipids, but modifications can be made to the neutral lipoplexes to increase entrapment efficiency. [67] Refer to Figure 1A for the general structure of lipoplexes.

\section{Lipopolyplexes}

Lipopolyplexes are liposomes that contain polymers; this siRNA carrier is advantageous because the lipid components of it are naturally biocompatible and have low immunogenicity while its polymeric components have a natural affinity for RNA. Studies have already shown that these lipopolyplexes not only demonstrate relatively reduced toxicity but also efficient delivery in organisms via inhalation. [16] Research on lipopolyplexes is currently being conducted to investigate their usefulness as an alternative to viral vectors for the treatment of Parkinson's Disease because it is stable, can cross the blood brain barrier, and target diseased brain cells specifically. [68] Refer to Figure 1B for the general structure of lipopolyplexes.

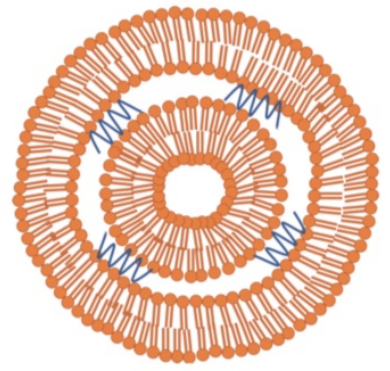

a. Lipoplexes

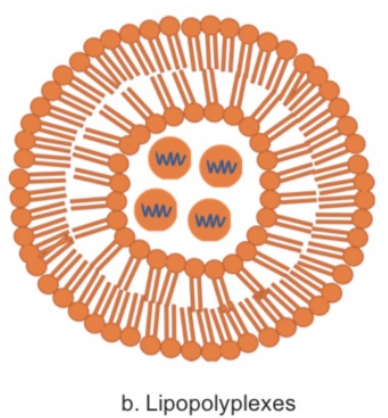

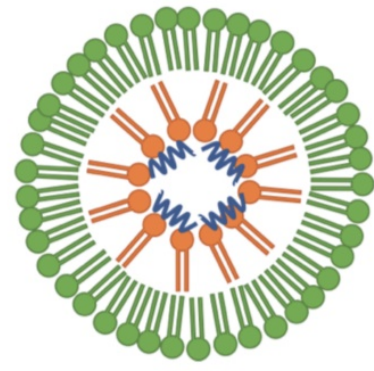

c. SNALPS

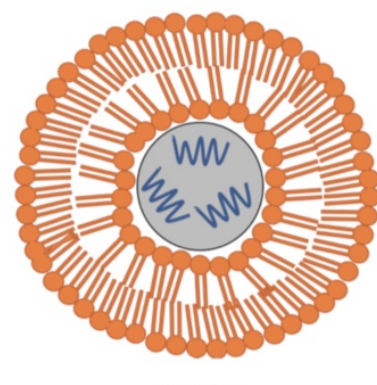

d. MCNPs
WW siRnA
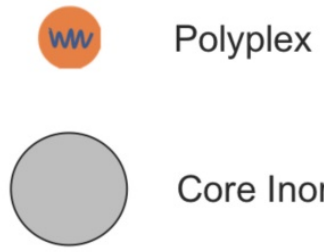

Core Inorganic NP

$=\quad$ Cationic Lipid

$=\quad$ Neutral Lipid

Figure 1. Lipid-based vectors for siRNA delivery can be generally classified into four different categories: lipoplexes, lipopolyplexes, SNALPs, and MCNPs. Adapted from Ref. [15]. 

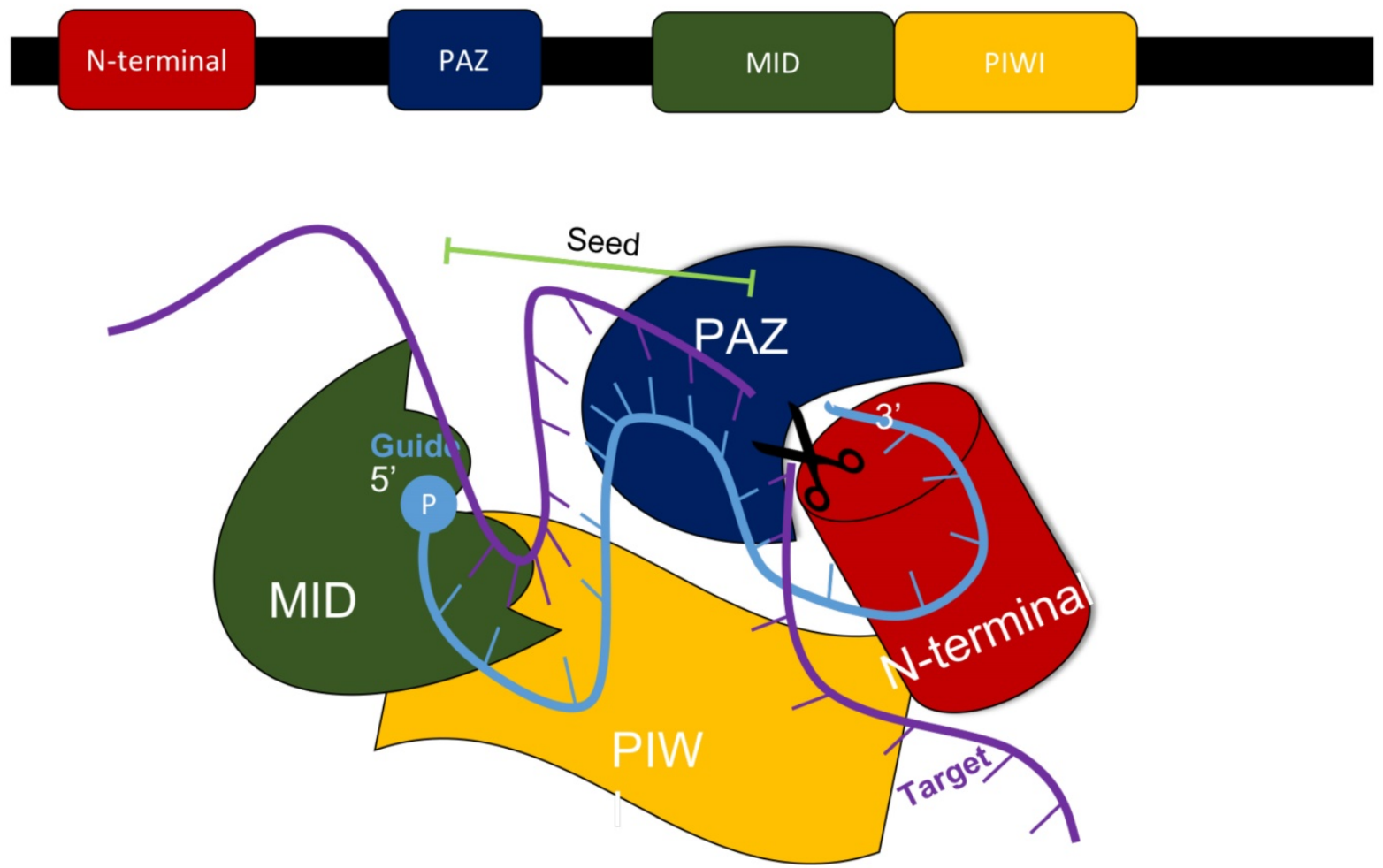

Figure 2. The MID, PIWI, PAZ, and N-terminal domains all are components of the AGO2 protein within the RISC complex that interacts with siRNA to silence the expression of a specific gene. Adapted from Ref. [3].
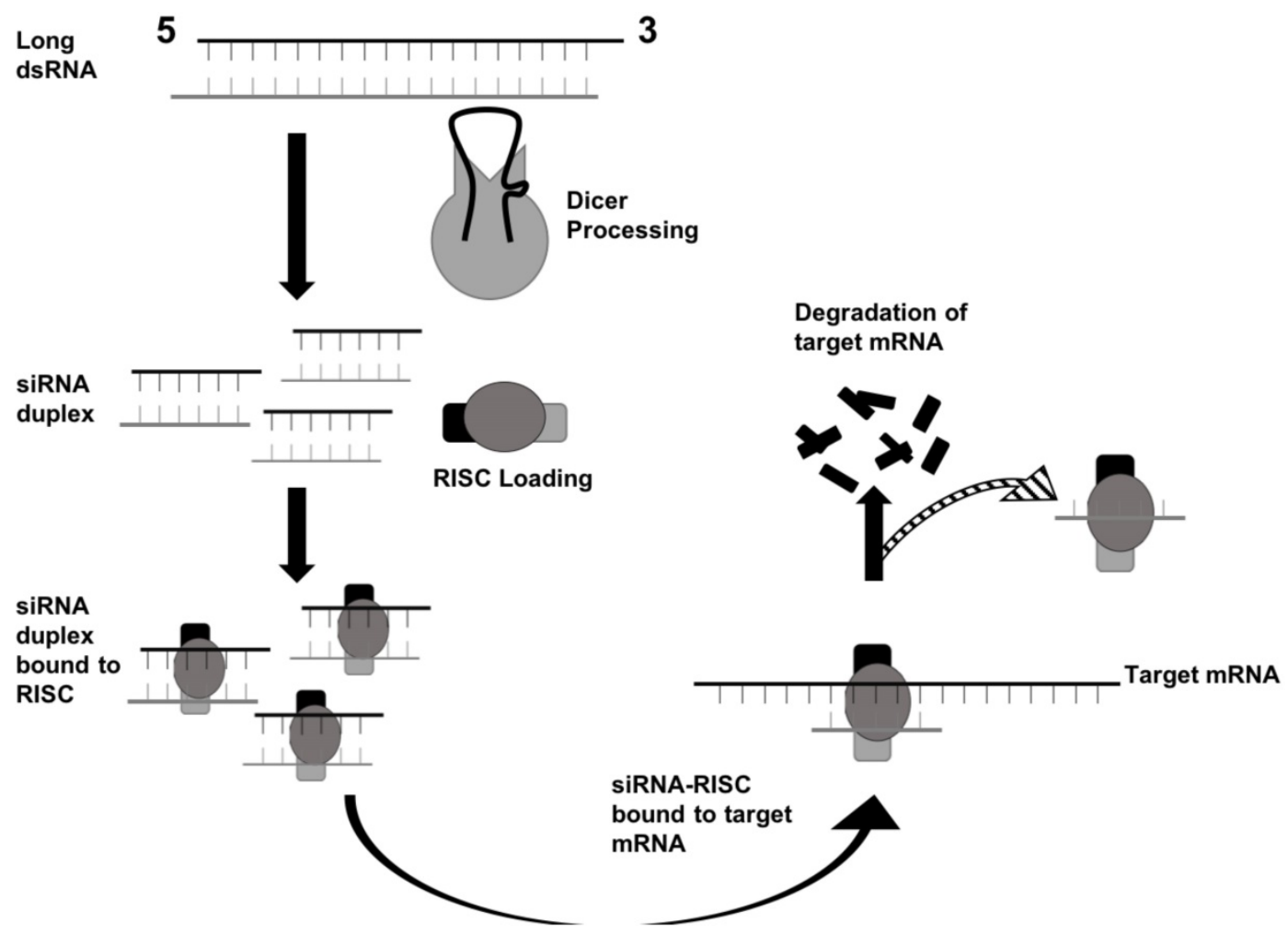

Figure 3. RNAi via siRNA begins with dsRNA being processed by Dicer. Then, the siRNA duplex interacts with RISC to degrade the target mRNA. Adapted from Ref. [30]. 


\section{SNALPs}

SNALPs differ from other liposomes due to the fact that they are composed of a single lipid bilayer containing an outer layer of neutral lipids and an inner layer of cationic lipids. The siRNA contained within SNALPs are typically found close to the inner membrane because of the attraction of the charges between the cationic lipids and the negatively charged siRNA. SNALPs also contain PEG-conjugated lipids. $[7,15,16]$ Polyethylene glycol, or PEG, can be added to lipid-based siRNA carriers so that the carriers would be able to circulate throughout the blood for an increased amount of time. The addition of PEG-conjugated lipids such as in SNALPs is one way to PEGylate lipid-based carriers. [17] PEGylation of liposomes leads to greater stability of the carrier. [18] Refer to Figure 1C for the general structure of SNALPs.

\section{MCNPs}

An MCNP is a siRNA carrier that has lipid bilayer surrounding one or more inorganic nanoparticles, which serve as the core. [15] One such inorganic nanoparticle could be calcium phosphate $(\mathrm{CaP})$ as it is not only biocompatible but is acid sensitive and can release its contents such as siRNA once in the cytoplasm of a cell. [20] Similar to MCNPs are liposome-polycation-DNA complexes (LPDs). LPDs that can help deliver siRNA are produced by mixing the appropriate siRNA with calf thymus DNA, which are then condensed with an arginine-rich, positively-charged polypeptide known as protamine. Afterwards, the core is then surrounded by liposomes. [18,21] Refer to Figure 1D for the general structure of an MCNP.

\section{Lipidoids}

Although they are not truly lipid-based, lipidoids are lipid-like molecules that could also aid in the delivery of siRNA. Studies in nonhuman primates and mice have already demonstrated that lipidoids can deliver functional siRNA for the mostly successful inhibition of the expression of certain genes while at the same time having low toxicity due to lower siRNA dosage. [7]

\section{Non-lipid Organic-Based Nanovectors}

\section{Chitosan}

One such non-lipid organic-based nanovector for siRNA delivery that researchers are currently investigating is chitosan. Chitosan is a cationic, linear polysaccharide that is made out of glucosamine/acetylglucosamine and has become an attractive vector for siRNA due to its biocompatibility, low cytotoxicity, and typical lack of immunogenicity. This polysaccharide is thus often considered to be Generally Recognized as Safe (GRAS). Furthermore, because chitosan is positively charged, it has the ability to interact with and protect negatively charged nucleic acids such as siRNA. [24,25] However, a significant challenge to chitosan research is its variability in composition and molecular weight, which makes it difficult to understand which properties are associated with which effect. [25] Chitosan transfection efficiency is sensitive to the $\mathrm{pH}$ of its surroundings, and as $\mathrm{pH}$ cannot be controlled in vivo, more research needs to be conducted to alleviate potential problems with the vector's transfection efficiency. Additionally, to optimize chitosan-delivery for siRNA, the ratio of the siRNA should be 5 to 10 times less than of the chitosan. [26] The amino group and the primary hydroxyl group of chitosan can be targeted for chemical modification so that the carrier can be enhanced for optimal siRNA delivery. $[27,28]$ Other modifications to chitosan such as PEGylation have also been demonstrated to optimize chitosan as a carrier. [29] Although chitosan still faces challenges, with more research and investigation, chitosan has great potential as a very effective vector for siRNA delivery.

\section{Dendrimers}

Another type of vector for siRNA delivery is called dendrimers, which are symmetric, highly branched macromolecules that begin with a core molecule and end at a functional chemical group. Similar to chitosan nanocarriers, dendrimers are biocompatible and have negligible immunogenicity; however, unlike unmodified chitosan, they are also water soluble. $[25,30,31]$ Dendrimers are also similar to lipid-based vectors due to high cytotoxicity being associated with not only cationic lipid-based vectors but cationic dendrimers as well. $[17,22,30]$ However, dendrimers can also be modified through PEGylation to not only increase the duration of its circulation in the blood but also to decrease its toxicity and increase its transfection efficacy. [32] The last/outer generation on the dendrimer (each concentric layer of a dendrimer is considered a generation) has a specific amount of functional groups that can be produced to ensure wanted interactions with appropriate tissues. [33] Dendrimers are synthesized synthetically in two ways: convergent and divergent synthesis. In divergent synthesis, dendrimers are synthesized by the addition of new generations around a multifunctional core (Figure 4), whereas in convergent synthesis, multiple dendrons are combined around the core to form a dendrimer. Downsides to each include the potential for branching 
defects in dendrimers synthesized divergently while dendrimers synthesized in a convergent manner have difficulty being truly spherical. [34,35] Two of the most successful and investigated dendrimers are polyamidoamine (PAMAM) and polypropyleneimine (PPI). Because PAMAM and PPI are amine-terminated, they are sensitive to $\mathrm{pH}$, which allows for increased specificity in the release of their contents. [35] Dendrimers are advantageous as siRNA carriers because researchers can strictly control its structure, especially in the number and size of the dendrimer's functional groups. [36]

\section{Polyethylenimines}

Polyethylenimines (PEIs) are another promising siRNA carrier. PEIs are cationic polymers that release its nucleic acid contents into a cell through the proton sponge effect in which PEIs promote endosomal escape by causing endosomal swelling and rupture due to osmolarity changes. Thus, this prevents lysosomal degradation. [37,38] The proton sponge effect is characteristic of PEIs due to their structure: every third atom is a protonable amino nitrogen atom. [39] PEIs also have two general structures: either linear (lPEI) or branched (bPEI). [37] PEIs have also demonstrated their usefulness as a siRNA carrier due to their successful delivery of siRNA molecules in animal models in vivo. [40] Furthermore, research on PEI modifications has already seen modified PEIs achieve reduced cytotoxicity while maintaining high efficacy. [41] However, in general, PEIs with higher molecular weights and bPEIs typically induce greater cytotoxicity compared to those with lower molecular weights and 1PEIs. PEIs are not easily biodegradable which leads to high toxicity, and although more research for PEI modifications to decrease cytotoxicity can be conducted, many researchers are simply exploring and studying other carriers that are associated with lower cytotoxicity. [42]

\section{Non-lipid Inorganic-Based Nanovectors}

\section{Gold Nanoparticles}

The use of gold nanoparticles (AuNPs) is a well-known inorganic vector for siRNA delivery as it is a safe and stable option due to its core being non-toxic, inert, and biocompatible. [43-45] The unique properties of the surface of AuNPs allow for this type of carrier to bond with siRNA covalently or non-covalently. When AuNPs and siRNA are bonded covalently, delivery can be further enhanced with the addition of a cationic polymer or cell penetrating peptides (CPPs). [43] Noncovalent AuNP-siRNA conjugates are also attractive because they provide options for the structural design of the carrier such as mixed-monolayer-protected AuNPs (MM-AuNPs) and amino acid-functionalized AuNPs (AA-AuNPs). [44] The versatility of AuNPs due to the ease of modifying its surface has made it an attractive carrier for siRNA delivery. [45] For example, it has been reported that AuNPs can be modified to have $\mathrm{pH}$-sensitivity so that the carriers could release their contents into a target region such as a slightly acidic tumor environment. [46] Furthermore, AuNPs are attractive due to their low cytotoxicity. [41] Additionally, AuNP conjugates have been reported to be successful in the topical delivery of siRNAs in vivo. $[41,47]$

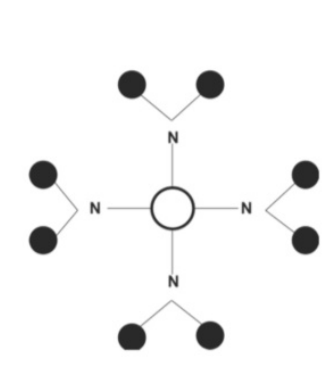

(A)

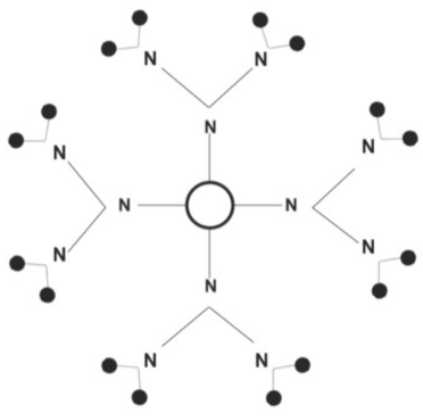

(B)

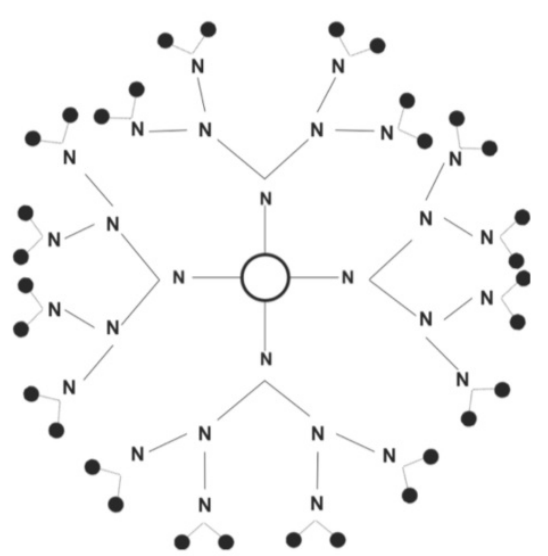

(C)

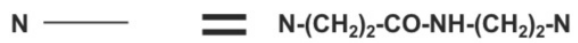

$\mathrm{N}-0 \mathrm{~N}-\left(\mathrm{CH}_{2}\right)_{2}-\mathrm{CO}-\mathrm{NH}-\left(\mathrm{CH}_{2}\right)_{2}-\mathrm{NH}_{2}$

Figure 4. A PAMAM dendrimer is synthesized in a divergent manner. Shown are (A) the first generation, (B) the second generation, and (C) the third generation of dendrimer. Adapted from Ref. [36]. 


\section{Superparamagnetic Iron Oxide Nanoparticles}

Biodegradability, biocompatibility, and nontoxicity are also characteristic of superparamagnetic iron oxide nanoparticles (SPIONs). The focus of current research on SPIONs includes their biodistribution and pharmacokinetics in order to better understand how they function as a carrier as well as to minimize any negative side effects such as toxicity. [48] The magnetic properties of SPIONs are size-dependent. The surface chemistry of SPIONs is another influential factor on their properties such as the extent of immune response they induce. [48-50] Therefore, research on different coatings for SPIONs has been conducted since a typical SPION has a hydrophobic surface and tend to agglomerate. Chitosan and PEG are two organic polymers that are used to help modify the surface chemistry of SPIONs. Other types of coating materials that are used are organic surfactants, inorganic metals, inorganic oxides, and bioactive molecules and structures. [49] Furthermore, because SPIONs are superparamagnetic, there is research that demonstrates how an external magnetic field could be applied for the selective delivery of SPIONs and thus causing an increase in transfection rates. [50] However, the use of SPIONs still requires more research as there is concern over the toxicity caused once the SPIONs are degraded in the body. [51]

\section{Mesoporous Silica-based Nanoparticles}

Another well-researched inorganic nanoparticle for siRNA delivery would be mesoporous silica-based nanoparticle (MSN). They are advantageous as a carrier because they have the ability to encapsulate more molecules compared to other carriers. Furthermore, MSNs are stable due to the iron oxide framework in their structure and have been reported to be able to escape the endosome for the release of its contents into the cytoplasm. [52] Additionally, controlled release is being investigated in MSNs because they can be chemically modified due to their stability and because MSN synthesis is relatively facile. [53] The synthesis of MSNs begins with one of two precursors: either tetraalkooxysilanes or sodium silicate solutions. Different templating agents such as ionic surfactants, pluronic surfactants, and neutral block copolymers are further used to influence pore size and arrangement as well the MSN's general shape and size. [54] The use of polyelectrolyte gatekeepers, supramolecularnanovalves, $\mathrm{pH}$-sensitive linkers, and acid-decomposable inorganic gatekeepers with MSNs to form a pH-responsive release system has also been studied to further enhance MSN delivery for the treatment of diseases such as cancer. [55] MSN-based systems have also been studied for the research of other stimuli-responsive delivery mechanisms for both endogenous stimuli such as enzymes and glucose and exogenous stimuli light and magnetics. [56] More research is still being conducted that focuses on the safety, biodegradability, pharmacokinetics, and biodistribution of MSNs. [54]

\section{Semiconductor Quantum Dots}

Semiconductor quantum dots have become attractive to researchers for siRNA delivery because of their usefulness in molecular imaging as fluorophores. As carriers, they exhibit biocompatibility. As methods of molecular imaging, quantum dots are attractive due to their relatively higher brightness, their smaller chances of photobleaching, and their ease in being detected compared to fluorescent dyes. $[41,46]$ Additionally, because of the tunable emission of quantum dots, they have become attractive to researchers who are studying the delivery of siRNA via these quantum dots into cells and small animals. [63]

\section{Cell-Penetrating Peptides}

Cell-penetrating peptides (CPPs) are small peptides that have the ability to carry siRNAs and other molecules and safely cross cell membranes. CPPs can also be referred to as protein transduction domains (PTDs). [57] Up to 30 amino acid residues can comprise one $\mathrm{CPP}$, of which many are arginine or lysine. [58] Although research is still being conducted concerning how CPPs enter cells to deliver their cargo, two major cellular uptake mechanisms are known: energy independent pathways and endocytosis. Examples of models that have been suggested for the latter include the inverted micelle, pore formation, carpet, and membrane thinning models. Studies have shown that cellular entry for CPPs is influenced by its properties as well as the properties of what it is delivering into the cell. [59] Interactions between CPPs and cell membranes are due to electrostatic interactions. Cationic CPPs have demonstrated high effectiveness in crossing anionic membranes. [60] CPPs can carry siRNA duplexes by either covalent or noncovalent linkage through electrostatic interactions or through the formation of disulfide bonds, respectively. Circumventing CPP entrapment in the lysosome has been investigated, resulting in the creation of methods to do so such as the addition of part of the hemagglutinin (HA) sequence originating from the human influenza virus to CPPs. [61] Organization of the multitude of CPPs varies depending on the what is used as the basis for 
their classification. Concerning their origin, CPPs can be considered to be protein-derived, chimeric, or synthetic. On the basis of their physicochemical characteristics, CPPs can be classified as cationic, amphipathic, or hydrophobic. [24,62] Challenges for the use of CPPs in siRNA delivery involve a potential decrease in delivery efficacy when positively-charged CPPs are linked to negatively-charged siRNA. Additionally, there is concern over CPPs having cytotoxicity or inducing immunogenicity. [7] However, CPPs are a promising carrier for siRNA delivery, and they continue to be investigated.

\section{Nanogels}

Nanogels are siRNA carriers that are composed of networks of either chemically or physically-linked polymers and are attractive as carriers due to their biocompatibility, high stability, and high loading capacity. Additionally, there is an ease to not only synthesizing nanogels but chemically modifying them as well. $[29,64]$ Preparing nanogels is simple, and neither mechanical energy nor organic solvents are required for their production. [64] Additional modifications of nanogels can aid in decreasing associated cytotoxicity. [65] Studies concerning nanogels in vivo have demonstrated their ability to protect their cargo from degradation. [66]

\section{siRNA and Carrier Modifications}

Modifications such as PEGylation have been briefly discussed with SNALPs. PEGylation allows carriers to circulate throughout the body for a longer duration as it prevents aggregation, opsonization, and phagocytosis as well as decreases the immunogenicity of its carrier. [69] Furthermore, PEG has been hailed as biocompatible and non-toxic. However, as research has advanced concerning PEGylation, limitations such as non-degradability have been demonstrated in PEGs, causing concerns over their usage. [70] Additionally, there have been reports of the PEGs themselves causing immunogenicity, especially with repeated usage in the same individual. [71] Thus, research has begun for finding alternatives to PEG such as with polysaccharides. [70,71]

Naked siRNA can also undergo chemical modifications to improve their effectiveness. Without modifications, naked siRNA face obstacles that will later be discussed in greater detail such as potential nuclease degradation, off-target effects, etc. [3,73] Additionally, modifying siRNA does not negatively affect their ability to silence target mRNA. Chemical modifications of siRNA have so far included modifications to the termini, backbone, sugars, and bases, but modifications have primarily focused on sugar moiety. The main goals of siRNA chemical modifications are to extend the half-life and to increase cellular uptake of the siRNA molecule. [74] Research has already established that modifying siRNAs with locked nucleic acids can extend its half-life to 90 hours without triggering severely negative effects, implying that RNAi machinery can tolerate a moderate amount of modifications to siRNA. Furthermore, to protect against siRNA degradation via nucleases, the backbone of the siRNA molecule can be altered. However, alterations can cause cytotoxicity as well as inhibit the silencing ability of siRNA. [3]

\section{Challenges and Applications for siRNA Therapy}

There have been many advances in the research of siRNA in RNAi and the differing techniques for their delivery. However, many obstacles still need to be overcome to maximize the advantages of siRNA therapy. Even as a multitude of siRNA carriers have been investigated and improved for the safe delivery of siRNA into cells, siRNA still risk not being able to undergo endosomal escape and enter the cytoplasm of the cells or even being degraded by RNAses within the cells as well. Additionally, there are risks of off-target silencing by the siRNA as well as even the activation of the body's immune system. [3] Off-target silencing can be either due to siRNA being able to tolerate a few mismatches in an mRNA transcript or due to siRNAs entering miRNA machinery that were already within the cell. In addition to these obstacles, there have also been reports of varying efficacies between siRNAs that targeted the same mRNA sequence, although different parts of the sequence. [5] Furthermore, even with the use of nanoparticles for the delivery of siRNA, researchers still face the challenges of developing siRNA carriers that can escape both the reticuloendothelial system and rapid renal clearance. It has therefore been suggested that nanoparticles should have a diameter of between $5-100 \mathrm{~nm}$ so that the carriers will have higher chances of avoiding both in the body. [72] As more research continues concerning the delivery of siRNA, researchers are finding newer and better ways to circumvent these obstacles and therefore enhance siRNA delivery. For example, modifications to both naked siRNA and siRNA carriers and their effects have been investigated, which were discussed previously in greater detail.

One prospective use for siRNA therapy is for the treatment of cancer. In cancer cells, apoptosis pathways are typically deregulated, but it is believed that siRNA can target the expression of anti-apoptosis 
genes so that apoptosis can be induced, killing the cancerous cells. Other genes siRNA can potentially target in cancer cells are the genes that are involved in signal transduction, angiogenesis, and drug resistance. [75] Research is also being conducted in the application of siRNA in the enhancement of bone regeneration and the treatment of musculoskeletal injuries and diseases. [76] Additional research is being invested in how siRNA can be used to treat ocular pathologies by targeting the molecules that contribute to the development of glaucoma, retinitis pigmentosa, and neovascular eye diseases. Although the use of siRNA therapy for diseases in the eyes have many advantages, this type of treatment can be further enhanced with more advancements in the development of controlled/targeted delivery techniques for siRNA to the eyes. [77] siRNAs can even potentially be used for the treatment of neurodegenerative diseases such as Alzheimer's, Parkinson's, and Huntington's disease. [78]

\section{Conclusion}

As more advancements in siRNA research are being made, siRNAs are becoming increasingly attractive for the treatment of a multitude of diseases including cancer as they can specifically target and downregulate the expression of certain genes by degrading their corresponsive mRNA transcripts. However, naked siRNA alone face many challenges within the body. Therefore, research is being intensively poured into advancing the development of safe and effective delivery systems for siRNA. Viral vectors are attractive as carriers for siRNA due to their high efficiency in delivery, which has directed much attention towards lentiviral vectors, adenoviral vectors, and adeno-associated vectors. However, viral vectors are associated with risks to biosafety such as high immunogenicity and potential insertional mutagenesis, which have raised many concerns among researchers. Therefore, non-viral vectors, although relatively less efficient compared to viral vectors, have thus become increasingly attractive for researchers because of their ease of production and relatively greater biosafety. However, prospective non-viral vectors for siRNA delivery should still be efficient and be able to target the appropriate cells. Furthermore, siRNA carriers must be able to circulate throughout the blood for an appropriate yet effective amount of time. High cellular uptake should be another characteristic of a good siRNA carrier. Although there are a variety of prospective non-viral vectors for siRNA delivery, there are three general categories used for the classification of non-viral vectors: lipid-based, non-lipid organic-based, and non-lipid inorganic based. The non-viral vectors that were discussed in this paper were lipoplexes, lipopolyplexes, stable nucleic-acid-lipid particles, membrane/core nanoparticles, lipidoids, chitosan, dendrimers, polyethylenimines, gold nanoparticles, superparamagnetic iron oxide particles, mesoporous silica-based nanoparticles, and semiconductor quantum dots. Other types of prospective carriers include cell penetrating peptides and nanogels. Each carrier has its own unique advantages and disadvantages, but most have the capacity to be modified or be used in conjunction with another carrier in order to maximize their beneficial characteristics. Modifications include the addition of polyethylene glycol and even direct alterations to naked siRNA molecules to further enhance siRNA delivery such as through controlled delivery. Additional modifications for other benefits are also being investigated, but researchers have to be careful to not inhibit the silencing ability of siRNA because of the modifications that they make. The use of RNA interference via siRNA for the treatment of diseases has great potential for the future, but there are still many obstacles to be overcome before the use of siRNA therapy becomes widespread. Research will thus continue as the use of siRNA has the potential to be applied to the treatment of many diseases such as cancer or even neurodegenerative diseases such as Alzheimer's disease. In fact, hybrid nanoparticles that are composed of more than one type of nanoparticles are being intensively researched to minimize the negative characteristics of single nanoparticles such as cytotoxicity. Hybrid nanoparticles can also function as a theranostic carrier and thus potentially aid in the monitoring of treatments and disease development. [79] Many advancements have been made that highlight the possibilities for siRNA therapy, and with continued research, many more advancements are sure to come.

\section{Competing Interests}

The authors have declared that no competing interest exists.

\section{References}

1. Lam J, Chow M, Zhang Y, Leung S. siRNA Versus miRNA as Therapeutics for Gene Silencing. Molecular Therapy. Nucleic Acids. 2015; 4: e252.

2. Guo W, Chen $W, Y u$ W, Huang W, Deng W. Small interfering RNA-based molecular therapy of cancers. Chinese Journal of Cancer. 2013; 32: 488-93.

3. Gavrilov K, Saltzman W. Therapeutic siRNA: Principles, Challenges, and Strategies. The Yale Journal of Biology and Medicine. 2012; 85: 187-200.

4. Zhang S, Zhi D, Huang L. Lipid-based vectors for siRNA delivery. Journal of Drug Targeting. 2012; 20:724-35.

5. Ozcan G, Ozpolat B, Coleman R, Sood A, Lopez-Berestein G. Preclinical and clinical development of siRNA-based therapeutics. Advanced Drug Delivery Reviews. 2015; 87: 108-19.

6. Maduri S. Applicability of RNA interference in cancer therapy: Current status. Indian J Cancer. 2015; 52: 11. 
7. Lee J, Yoon T, Cho Y. Recent Developments in Nanoparticle-Based siRNA Delivery for Cancer Therapy. BioMed Research International. 2013; 2013: 1-10.

8. Li J, Wang Y, Zhu Y, Oupický D. Recent advances in delivery of drug-nucleic acid combinations for cancer treatment. Journal of Controlled Release : Official Journal of the Controlled Release Society. 2013; 172: 589-600.

9. Hutson T, Foster E, Moon L, Yáñez-Muñoz R. Lentiviral Vector-Mediated RNA Silencing in the Central Nervous System. Human Gene Therapy Methods. 2014; 25:14-32

10. Wold W, Toth K. Adenovirus Vectors for Gene Therapy, Vaccination and Cancer Gene Therapy. Current Gene Therapy. 2014; 13: 421-33.

11. Borel F, Kay M, Mueller C. Recombinant AAV as a Platform for Translating the Therapeutic Potential of RNA Interference. Molecular Therapy. 2014; 22: 692-701.

12. Zinn E, Vandenberghe L. Adeno-associated virus: fit to serve. Current Opinion in Virology. 2014; 8: 90-7.

13. Trapani I, Puppo A, Auricchio A. Vector platforms for gene therapy of inherited retinopathies. Progress in Retinal and Eye Research. 2014; 43: 108-28.

14. Chira S, Jackson C, Oprea I et al. Progresses towards safe and efficient gene therapy vectors. Oncotarget. 2015; 6: 30675-703.

15. Xia Y, Tian J, Chen X. Effect of surface properties on liposomal siRNA delivery. Biomaterials. 2016; 79: 56-68.

16. Xue $\mathrm{H}$, Guo P, Wen W, Wong H. Lipid-Based Nanocarriers for RNA Delivery. Current Pharmaceutical Design. 2015; 21: 3140-7.

17. Immordino M, Dosio F, Cattel L. Stealth liposomes: review of the basic science, rationale, and clinical applications, existing and potential. International Journal of Nanomedicine. 2006; 1: 297-315.

18. Zhang J, Li X, Huang L. Non-viral nanocarriers for siRNA delivery in breast cancer. Journal of Controlled Release: Official Journal of the Controlled Release Society. 2014; 190: 440-50.

19. Mashaghi S, Jadidi T, Koenderink G, Mashaghi A. Lipid Nanotechnology. International Journal of Molecular Sciences. 2013; 14: $4242-82$

20. Guo S, Huang L. Nanoparticles containing insoluble drug for cancer therapy. Biotechnology Advances. 2014; 32: 778-88.

21. Zhang Y, Satterlee A, Huang L. In Vivo Gene Delivery by Nonviral Vectors: Overcoming Hurdles?. Molecular Therapy. 2012; 20: 1298-304.

22. Yurgel V, Collares T, Seixas F. Developments in the use of nanocapsules in oncology. Brazilian Journal of Medical and Biological Research. 2013; 46: 486-501

23. Sercombe L, Veerati T, Moheimani F, Wu S, Sood A, Hua S. Advances and Challenges of Liposome Assisted Drug Delivery. Frontiers in Pharmacology. $2015 ; 6 ; 286$

24. Layek B, Lipp L, Singh J. Cell Penetrating Peptide Conjugated Chitosan for Enhanced Delivery of Nucleic Acid. International Journal of Molecular Sciences. 2015; 16: 28912-30.

25. Bellich B, D'Agostino I, Semeraro S, Gamini A, Cesàro A. "The Good, the Bad and the Ugly" of Chitosans. Marine Drugs. 2016; 14: 99.

26. Raftery R, O'Brien F, Cryan S. Chitosan for Gene Delivery and Orthopedic Tissue Engineering Applications. Molecules. 2013; 18: 5611-47.

27. Zhang J, Xia W, Liu $\mathrm{P}$ et al. Chitosan Modification and Pharmaceutical/Biomedical Applications. Marine Drugs. 2010; 8: 1962-87.

28. Patel M, Patel R, Patel J. Chitosan Mediated Targeted Drug Delivery System: A Review. Journal of Pharmacy \& Pharmaceutical Sciences. 2010; 13: 536-57.

29. Singha K, Namgung $R$, Kim W. Polymers in Small-Interfering RNA Delivery. Nucleic Acid Therapeutics. 2011; 21: 133-47.

30. Wang J, Lu Z, Wientjes M, Au J. Delivery of siRNA Therapeutics: Barriers and Carriers. The AAPS Journal. 2010; 12: 492-503.

31. Draz M, Fang B, Zhang P et al. Nanoparticle-Mediated Systemic Delivery of siRNA for Treatment of Cancers and Viral Infections. Theranostics. 2014; 4: 872-92.

32. Biswas S, Torchilin V. Dendrimers for siRNA Delivery. Pharmaceuticals. 2013; 6: 161-83

33. Kannan R, Nance E, Kannan S, Tomalia D. Emerging concepts in dendrimer-based nanomedicine: from design principles to clinical applications. Journal of Internal Medicine. 2014; 276: 579-617.

34. Bugno J, Hsu H, Hong S. Tweaking dendrimers and dendritic nanoparticles for controlled nano-bio interactions: potential nanocarriers for improved cancer targeting. Journal of Drug Targeting. 2015; 23: 642-50.

35. Kesharwani $\mathrm{P}$, Iyer $\mathrm{A}$. Recent advances in dendrimer-based nanovectors for tumor-targeted drug and gene delivery. Drug Discovery Today. 2015; 20: $536-47$.

36. Xu Q, Wang C, Wayne Pack D. Polymeric Carriers for Gene Delivery: Chitosan and Poly(amidoamine) Dendrimers. Current Pharmaceutical Design. 2010; 16: 2350-68.

37. Jin L, Zeng X, Liu M, Deng $Y$, He N. Current Progress in Gene Delivery Technology Based on Chemical Methods and Nano-carriers. Theranostics. 2014; 4: 240-55.

38. Koirala A, Conley S, Naash M. A review of therapeutic prospects of non-viral gene therapy in the retinal pigment epithelium. Biomaterials. 2013; 34: 7158-67.

39. Boussif $\mathrm{O}$, Lezoualc'h $\mathrm{F}$, Zanta $\mathrm{M}$ et al. A versatile vector for gene and oligonucleotide transfer into cells in culture and in vivo: polyethylenimine. Proceedings of the National Academy of Sciences of the United States of America. 1995; 92: 7297-301.

40. Zhang Y, Wang Z, Gemeinhart RA. Progress in MicroRNA Delivery. Journal of Controlled Release : Official Journal of the Controlled Release Society. 2013; 172: 962-74.

41. Kozielski KL, Tzeng SY, Green JJ. Bioengineered Nanoparticles for siRNA delivery. Wiley Interdisciplinary Reviews. Nanomedicine and Nanobiotechnology. 2013; 5: 449-68

42. Xue HY, Liu S, Wong HL. Nanotoxicity: a key obstacle to clinical translation of siRNA-based nanomedicine. Nanomedicine (London, England). 2014; 9: 295-312.

43. Jiang Y, Huo S, Hardie J, Liang XJ, Rotello VM. Progress and perspective of inorganic nanoparticles based siRNA delivery system. Expert Opinion on Drug Delivery. 2016; 13:547-59.

44. Ding Y, Jiang Z, Saha K, Kim CS, Kim ST, Landis RF, Rotello VM. Gold Nanoparticles for Nucleic Acid Delivery. Molecular Therapy. 2014; 22:1075-83.

45. Rana S, Bajaj A, Mout R, Rotello VM. Monolayer coated gold nanoparticles for delivery applications. Advanced Drug Delivery Reviews. 2012; 64: 200-16.

46. Wang Z, Liu G, Zheng H, Chen X. Rigid nanoparticle-baseddelivery of anti-cancer siRNA: challenges and opportunities. Biotechnology Advances. 2014; 32:831-43

47. Zheng D, Giljohann DA, Chen DL, Massich MD, Wang XQ, Iordanov $\mathrm{H}$, et al. Topical delivery of siRNA-based spherical nucleic acid nanoparticle conjugates for gene regulation. Proceedings of the National Academy of Sciences of the United States of America. 2012; 109: 11975-80.

48. Arami $\mathrm{H}$, Khandhar A, Liggitt D, Krishnan KM. In vivo delivery, pharmacokinetics, biodistribution and toxicity of iron oxide nanoparticles. Chemical Society Reviews. 2015; 44: 8576-607.

49. Krishnan KM. Biomedical Nanomagnetics: A Spin Through Possibilities in Imaging, Diagnostics, and Therapy. IEEE Transactions on Magnetics. 2010; 46: 2523-58

50. Shubayev VI, Pisanic TR, Jin S. Magnetic nanoparticles for theragnostics. Advanced Drug Delivery Reviews. 2009; 61: 467-77.

51. Thomsen LB, Thomsen MS, Moos T. Targeted drug delivery to the brain using magnetic nanoparticles. Therapeutic Delivery. 2015; 6: 1145-55.

52. Gandhi NS, Tekade RK, Chougule MB. Nanocarrier mediated Delivery of siRNA/miRNA in Combination with Chemotherapeutic Agents for Cancer Therapy: Current Progress and Advances. Journal of Controlled Release: Official Journal of the Controlled Release Society. 2014; 194: 238-56.

53. Mekaru H, Lu J, Tamanoi F. Development of mesoporous silica-based nanoparticles with controlled release capability for cancer therapy. Advanced Drug Delivery Reviews. 2015; 95: 40-9.

54. Roggers R, Kanvinde S, Boonsith S, Oupický D. The Practicality of Mesoporous Silica Nanoparticles as Drug Delivery Devices and Progress Toward This Goal. AAPS PharmSciTech. 2014; 15: 1163-71.

55. Yang KN, Zhang CQ, Wang W, Wang PC, Zhou JP, Liang XJ. pH-responsive mesoporous silica nanoparticles employed in controlled drug delivery systems for cancer treatment. Cancer Biology \& Medicine. 2014; 11: 34-43.

56. Song $\mathrm{Y}, \mathrm{Li} \mathrm{Y}, \mathrm{Xu} \mathrm{Q}$, Liu Z. Mesoporous silica nanoparticles for stimuli-responsive controlled drug delivery: advances, challenges, and outlook. International Journal of Nanomedicine. 2017; 12: 87-110.

57. Zahid M, Robbins P. Cell-Type Specific Penetrating Peptides: Therapeutic Promises and Challenges. Molecules. 2015; 20: 13055-70.

58. Ruczynski J, Wierzbicki PM, Kogut-Wierzbicka M, Mucha P, Siedlecka-Kroplewska K, Rekowski P. Cell-penetrating peptides as a promising tool for delivery of various molecules into the cells. Folia Histochemica et Cytobiologica. 2015; 52: 257-69.

59. Shin MC, Zhang J, Min KA, Lee K, Byun Y, David AE, et al. Cell-penetrating peptides: achievements and challenges in application for cancer treatment. Journal of Biomedical Materials Research. Part A. 2014; 102: $575-87$.

60. Kauffman WB, Fuselier T, He J, Wimley WC. Mechanism Matters: A Taxonomy of Cell Penetrating Peptides. Trends in Biochemical Sciences. 2015; 40: 749-64.

61. Huang YW, Lee HJ, Tolliver LM, Aronstam RS. Delivery of Nucleic Acids and Nanomaterials by Cell-Penetrating Peptides: Opportunities and Challenges. BioMed Research International. 2015; 2015: 834079.

62. Chérine B, Sandrine S. Cell-penetrating peptides: 20 years later, where do we stand? FEBS Letters. 2013; 587: 1693-702.

63. Zhao MX, Zhu BJ. The Research and Applications of Quantum Dots as Nano-Carriers for Targeted Drug Delivery and Cancer Therapy. Nanoscale Research Letters. 2016; 11: 207.

64. Soni G, Yadav KS. Nanogels as potential nanomedicine carrier for treatment of cancer: A mini review of the state of the art. Saudi Pharmaceutical Journal: SPJ. 2016; 24: 133-9.

65. Chacko RT, Ventura J, Zhuang J, Thayumanavan S. Polymer nanogels: a versatile nanoscopic drug delivery platform. Advanced Drug Delivery Reviews. 2012; 64: 836-51.

66. Vinogradov SV. Nanogels in the race for drug delivery. Nanomedicine. 2010;5: 165-8.

67. Wu SY, McMillan NAJ. Lipidic Systems for In Vivo siRNA Delivery. The AAPS Journal. 2009; 11: 639-52. 
68. Chen W, Li H, Liu Z, Yuan W. Lipopolyplex for Therapeutic Gene Delivery and Its Application for the Treatment of Parkinson's Disease. Frontiers in Aging Neuroscience. 2016; 8: 68.

69. Suk JS, Xu Q, Kim N, Hanes J. Ensign LM. PEGylation as a strategy for improving nanoparticle-based drug and gene delivery. Advanced Drug Delivery Reviews. 2016; 99(Pt A): 28-51.

70. Qi Y, Chilkoti A. Protein-Polymer Conjugation-Moving Beyond PEGylation. Current Opinion in Chemical Biology. 2015; 28: 181-93.

71. Zhang F, Liu M, Wan H. Discussion about Several Potential Drawbacks of PEGylated Therapeutic Proteins. Biological and Pharmaceutical Bulletin. 2014; 37: 335-9.

72. Miele E, Spinelli GP, Miele E, Di Fabrizio E, Ferretti E, Tomao S, Gulino A. Nanoparticle-based delivery of small interfering RNA: challenges for cancer therapy. International Journal of Nanomedicine. 2012; 7: 3637-57.

73. Peacock H, Kannan A, Beal PA, Burrows CJ. Chemical Modification of siRNA Bases to Probe and Enhance RNA Interference. The Journal of Organic Chemistry. 2011; 76: 7295-300.
74. Yang C, Zhang C, Zhao Z, Zhu T, Yang B. Fighting against kidney diseases with small interfering RNA: opportunities and challenges. Journal of Translational Medicine. 2015; 13: 39

75. Guo P, Coban O, Snead N, Trebley J, Hoeprich S, Guo S, Shu Y. Engineering RNA for Targeted siRNA Delivery and Medical Application. Advanced Drug Delivery Reviews. 2010; 62: 650-66.

76. Ghadakzadeh S, Mekhail M, Aoude A, Hamdy R, Tabrizian M. Small Players Ruling the Hard Game: siRNA in Bone Regeneration. Journal of Bone and Mineral Research. 2016; 31: 475-87.

77. Guzman-Aranguez A, Loma P, Pintor J. Small-interfering RNAs (siRNAs) as a promising tool for ocular therapy. British Journal of Pharmacology. 2013; 170: 730-47.

78. Reddy LV, Miller TM. RNA-targeted Therapeutics for ALS. Neurotherapeutics. 2015; 12: 424-7.

79. Sailor MJ, Park JH. Hybrid Nanoparticles for Detection and Treatment of Cancer. Advanced Materials (Deerfield Beach, Fla.). 2012; 24: 3779-802. 